\title{
Prácticas instituyentes en el espacio vecinal: el barrio como un común
}

\author{
Francisco Letelier
}

CEUT Universidad Católica del Maule, Talca, Chile. Email: fletelier@ucm.cl

\section{Stefano Micheletti}

CEUT Universidad Católica del Maule, Talca, Chile.

Email: smicheletti@ucm.cl

\section{Julien Vanhulst}

CEUT Universidad Católica del Maule, Talca, Chile.

Email: julien@ucm.cl

\begin{abstract}
Resumen $^{1}$ : El presente artículo propone una reflexión acerca de las posibilidades de concebir el barrio como un “común” (bien común, espacio común) a través de las prácticas y acciones colectivas que producen la vida barrial y que simultáneamente instituyen un orden político particular. Se sostiene que gran parte de la producción de este espacio se hace fuera del ámbito del mercado y de la regulación pública centralizada y que se materializa por medio de las prácticas de los residentes. Estas pueden expresarse en actos cotidianos y de pequeña escala o en acciones de mayor alcance y articulación, que permiten hablar de gobernanza del territorio como común. En un contexto en que la capacidad de cogestión ampliada está marginada por los efectos de una modernización neoliberal y sus políticas urbanas, abrimos una discusión sobre los bienes comunes, el principio de lo común y el lugar del barrio bajo este marco conceptual. También revisamos — como caso de estudio - una experiencia local en que, al aumentar la escala de articulación vecinal, se logra fortalecer las capacidades de acción colectiva, posibilitándose con ello el inicio de un proceso de gobernanza ampliada.
\end{abstract}

Palabras clave: barrio, bien común, reproducción social.

\section{Institutive practices in the local space: the neighborhood as a common}

\begin{abstract}
This article proposes a reflection about the possibilities of conceiving the neighborhood as a "common" (common good, common space) through practices and collective actions that produce neighborhood life and simultaneously institute a particular political order. It is argued that much of the production of this space occurs outside of the spheres of the market and centralized government regulation, and is embodied through residents' practices. These can be expressed in small-scale everyday actions or in those of a greater scope whose articulation allows one to speak of governance as a common territory. In a context where the ability for self-management is marginalized by
\end{abstract}


the effects of neoliberal modernization and urban policies, we open a discussion about the commons and the role of the neighborhood in this framework. We also reviewed a local experience in which increasing the scale of neighborhood strengthened the capacity for collective action, allowing thus the beginning of a process of extended governance.

Keywords: Common good, neighborhood, social reproduction.

\section{Práticas instituintes no espaço vicinal: o bairro como um comum}

Resumo: O presente artigo propõe uma reflexão a respeito das possibilidades de conceber o bairro como um “comum” (bem comum, espaço comum) através das práticas e ações coletivas que produzem a vida no bairro, e que simultaneamente instituem uma ordem política particular. Se sustenta que grande parte da produção deste espaço se faz fora do âmbito do mercado e da regulação pública centralizada, e que materializa-se por meio das práticas dos residentes. Estas podem expressar-se em atos quotidianos e de pequena escala, ou em ações de maior alcance e articulação que permitem falar de governança do território como algo comum. Num contexto em que a capacidade de cogestão ampliada está marginada pelos efeitos de uma modernização neoliberal e suas políticas urbanas, abrimos uma discussão sobre os bens comuns, o principio do comum e o lugar do bairro sob este marco conceitual. Também revisamos como estudo de caso - uma experiência local em que, ao aumentar a escala de articulação vicinal, se consegue fortalecer as capacidades de ação coletiva, possibilitando-se com isso o inicio de um processo de governança ampliada.

Palavras-chaves: bairro, bem comum, reprodução social.

$* * *$

\section{Introducción}

Más allá del restringido margen de los programas focalizados de mejoramiento barrial y de las escuálidas políticas de participación comunitaria de los gobiernos democráticos ${ }^{2}$, desde los años noventa han venido resurgiendo en Chile movimientos de pobladores que orientan su acción hacia la reivindicación del derecho a la ciudad y no solo al mejoramiento de sus condiciones básicas de vida a través del acceso a vivienda, a tierra urbana y a servicios. Desde el año 2000, por otra parte, se han generado también nuevos movimientos o agrupaciones de clase media y medio-alta, que orientan su acción colectiva hacia la defensa de sus estilos de vida, cada vez más amenazados por la irrupción del mercado y la incapacidad del Estado de velar por el bien común (Márquez, 2009). En diversos contextos se producen prácticas vecinales que superan largamente los estrechos márgenes de los programas focalizados y de la legislación: mesas territoriales, fiestas y ferias vecinales, cabildos, comités ambientales, mingas urbanas, diagnósticos participativos, mapeos vecinales, entre otras muchas. Este conjunto de experiencias, que constituyen componentes centrales de la producción cotidiana del barrio, están fuera del campo de visión de la acción del Estado y en 
muchos casos son debilitadas por las propias políticas urbanas.

En este contexto, este artículo sostiene que se requiere de nuevos enfoques para abordar la escala barrial y que ellos deben tener una perspectiva más amplia que la de generar condicione urbanas de gobernabilidad para mitigar los daños colaterales del urbanismo neoliberal (Bauman, 2011). Estos nuevos enfoques deben permitir que fluyan y se fortalezcan las múltiples formas de acción de las comunidades y organizaciones en el territorio, que ellas sean visibles para el Estado y, sobre todo, para los propios ciudadanos. Que sean respetadas y estimuladas.

¿Por qué hacer hincapié en las relaciones basadas en el territorio, cuando muchos sostienen que la vida actual estaría fuertemente desterritorializada? Porque, como sostiene Arendt (1993), el origen del poder reside en la contigüidad, en la vecindad: “solo donde los hombres viven tan unidos que las potencialidades de la acción están siempre presentes, el poder puede permanecer con ellos” (p. 224); y porque, como sostiene Lefebvre (1978), la contigüidad vecinal es irremplazable: "relaciones de consanguineidad, contigüidad y vecindad, que durante tantos siglos fueron sostén y encanto, ambiguos y limitados, pero poderosos, de la existencia social, todas estas relaciones se desmoronan. Y nada las reemplaza” (p. 180).

Es necesario introducir nuevos equilibrios en la forma en que se construye lo barrial, y eso requiere relevar el rol de la dimensión común por sobre la estatal y la privada. Falta, como sostiene Óscar Landerretche en su libro “Vivir juntos (2016), una ‘política urbana comunitaria””. Proponemos reflexionar sobre el barrio en su dimensión de bien común. Este enfoque implica visibilizar y potenciar las acciones de producción social de las propias comunidades y reconocerlas como parte sustantiva de la recreación permanente de la vida vecinal en tanto "praxis instituyente” (Castoriadis, 1975), expresada en procesos económicos que se encuentran fuera de lo mercantil y en prácticas sociopolíticas que resignifican el barrio y el convivir cotidiano.

Desde esta perspectiva es que revisamos un caso en que vecinos y vecinas de tres barrios de la ciudad de Talca, en la zona central de Chile, apoyados por un consorcio de dos ONG y una universidad regional, han emprendido un trabajo de articulación territorial y construcción colectiva de agendas de desarrollo barrial. Los resultados de esta experiencia muestran con claridad que, pese a las dificultades, el fortalecimiento de la capacidad de acción colectiva de las comunidades y sus organizaciones instituye nuevos mecanismos y significados en la relación con el territorio y con el Estado, y abren la puerta para que, a partir de estas nuevas relaciones, se fortalezca la dimensión común de los barrios. 


\section{Los bienes comunes y la producción social del espacio barrial}

\section{El barrio como un común}

Los bienes comunes (en sentido amplio: materiales o inmateriales, y servicios) pueden ser definidos como los recursos que son movilizados por una acción colectiva con una finalidad de interés compartido. Incorporan generalmente la idea de una construcción simultánea de un bien y de una comunidad, en pos de la sustentabilidad socioambiental. Su modo de producción y/o de gestión (por la acción colectiva) los distingue de los bienes privados (producidos por los individuos para ellos mismos o para el mercado) y de los públicos (producidos y/o regulados por la esfera pública). Fundamentalmente, el concepto nos invita a superar una definición económica (artefacto de producción o de consumo) para cuestionar nuestro sentido filosófico y político de lo común (Laval \& Dardot, 2014; Ostrom, 2010, 2011). Permite reconocer la riqueza creada socialmente y nombrar las prácticas colectivas que les dan forma y vida, abriendo el estrecho marco de las finalidades de la acción cercado por la lógica capitalista. Estas prácticas no pueden ser clasificadas en la categoría de las actividades mercantiles ni de la acción pública tradicional (lo que no impide que se interconecten).

Podemos mencionar que distintos tipos de bienes comunes (urbanos, ambientales, económicos, genéticos, de conocimiento, etc.) conocen semejante gobernanza y resguardan diferentes aspectos de una nueva prosperidad: sustentabilidad socioecológica, importancia del vínculo social y la calidad de vida, experiencias participativas, aprendizaje y acción colectiva, movilización de formas de acción política hibridas que implican simultáneamente la esfera pública, las comunidades y las redes de ciudadanos.

Siguiendo esta propuesta, los bienes que entran en esta categoría se refieren a una gran diversidad de recursos, respecto de los cuales no existe un inventario exhaustivo. Los comunes están caracterizados por una amplia variedad de modos de producción y de gestión, y se constituyen como tales movilizando una acción colectiva que proviene de una comunidad o de una red de ciudadanos que deciden en común su modo de producción o de gestión.

Ejemplos de nuevas modalidades para la gestión urbana —que comparten el principio de la coactividad en la construcción de valor comúnson las cooperativas de vivienda y los proyectos de Community land trust que partieron inicialmente en Inglaterra y Estados Unidos, y que apuntan a quitar las tierras del mercado de la construcción tradicional, para evitar la especulación sobre la tierra y promover el derecho a la vivienda. Otros casos interesantes a nivel internacional son el Urbanismo P2P (Peer-topeer: entre pares, de igual a igual), que nace de una nueva generación de investigadores y colectivos que proponen un diseño de ciudad a escala humana; y el Movimiento Ciudades en Transición, que apunta al desarrollo 
de iniciativas a favor del consumo de bienes de producción local y/o colectiva, la agroecología, la permacultura, el decrecimiento, etc. En Chile podemos mencionar los casos de la agrupación Vecinos por la Defensa del Barrio Yungay, y de Ciudad Vida, una red de organizaciones que en Santiago trabaja para dar a las comunidades urbanas un rol más activo en la planificación, a fin de construir barrios y ciudades más justas socialmente y sustentables ambientalmente. También es relevante el caso del Programa Territorio y Acción Colectiva que se desarrolla en Talca, y que abordaremos en profundidad más adelante.

Elinor Ostrom (2010 y 2011) ha mostrado en su trabajo de más de cincuenta años que muchos colectivos en el mundo lograron proteger ciertos comunes contra una visión neoliberal exclusiva. Según la autora, un bien es común solo cuando está manejado/gobernado de manera compartida bajo reglas que la comunidad se da. En este marco, ha identificado algunos aspectos centrales para este tipo de gestión, que dicen relación con la necesidad de establecer límites y accesos al recurso claramente identificados, con la definición de espacios de deliberaciones colectivas, la existencia de reglas claras y de mecanismos de resolución de conflictos , así como el reconocimiento del derecho de auto organización.

Así, lo común no se encuentra en la esencia de las cosas o de los bienes, sino en una filosofía pragmática que opone una "praxis instituyente" (Castoriadis, 1975) a la perspectiva estructuralista de la dominación de la institución. Bajo esta perspectiva, ninguna institución de lo común esta preestablecida ni es inmutable y existe una multitud de bienes comunes invisibilizados porque no están reconocidos en el discurso social dominante, pero que, sin embargo, participan en la reproducción de la sociedad.

Saliendo del campo económico y de la propuesta de la gobernanza de los comunes, después de Ostrom (2010, 2011), Christian Laval y Pierre Dardot (2014) proponen llevar la idea de lo común al campo político y pasar de la noción de bien común a un principio político de lo común. Según estos autores lo común hace referencia a la posibilidad de sustraer un bien a la propiedad privada y a la propiedad pública, para practicar una gestión que beneficia a todos los miembros de la comunidad. Se introducen así dos aspectos de gobernanza (en línea con Ostrom y Castoriadis): por un lado, rechazar la identificación de lo común con lo que es público (en el sentido de propiedad pública o estatal); y, por otro lado, definirlo a partir de una actividad o acción común. Así, la tesis de Laval y Dardot (2014) es que "lo común debe ser pensado como co-actividad, y no como co-pertenencia, copropriedad o co-posesión” (p. 48); ello considerando que la pertenencia o propiedad está supeditada a la coactividad y, por consiguiente, no es el eje articulador de las relaciones.

En síntesis, y cruzando las propuestas de Ostrom, Castoriadis y de Laval y Dardot, lo común es el resultado de una praxis instituyente, que nace en el "magma ${ }^{3}$ de los imaginarios sociales" (Castoriadis, 1975), una coactividad (Laval y Dardot) que crea nuevas instituciones y formas de 
gobernanza (nuevos arreglos institucionales, según Ostrom). Por lo mismo, lo común no se puede definir de manera "transhistorica” (no hay bienes comunes per se). De lo anterior se desprende la importancia de visibilizar los procesos en que los propios sujetos, colectiva y cotidianamente, producen socialmente su barrio e instituyen nuevos arreglos institucionales y nuevos tipos de relación con el territorio, la colectividad, y con lo público y lo privado.

\section{La producción social del espacio barrial como un común}

El barrio es producto de un amplio conjunto de prácticas y dinámicas sociales y económicas. Algunas de ellas operan dentro del espacio del mercado, otras en la esfera pública y unas terceras en el ámbito social y comunitario. Denominamos a estas últimas 'prácticas de reproducción social del barrio’ (PRSB), y las consideramos la base desde donde pensar el barrio como un común. Tal como señala Delamaza (2016), en la medida en que el barrio empieza a pensar como colectivo y permite que varios confluyan para acometer una propuesta de abordaje de la inversión y de transformación integral, el Estado es desafiado. De hecho, existen experiencias muy interesantes de coordinación alrededor de distintos consejos de desarrollo para decidir cómo invertir recursos públicos, superando la lógica de la planificación sectorial de las políticas. Cuando esto sucede, la acción comunitaria, en tanto fuerza de reproducción ampliada del territorio, comienza a orientar la acción de la economía pública.

Si cuando hablamos de reproducciónsimple nos referimos al trabajo del conjunto de unidades domésticas para lograr la reproducción biológica y cultural de sus miembros (Coraggio, 1999), cuando asistimos a un proceso en que, por encima del nivel de reproducción simple, se verifica durante un período prolongado un desarrollo sostenido en la calidad de vida de los miembros de una unidad doméstica, hablamos de reproducción ampliada. En este caso, las unidades domésticas generan extensiones de su lógica de reproducción mediante asociaciones, comunidades organizadas, redes formales o informales de diverso tipo, consolidando organizaciones socioeconómicas dirigidas a mejorar las condiciones de reproducción de la vida de sus miembros (Coraggio, 1999). La vida barrial, entonces, se reproduce de manera comunitaria, vecinal, más allá de la familia y de las relaciones de parentesco. Pero estas acciones son invisibles al ojo de los economistas. La teoría económica convencional expresa fundamentalmente lo que pasa en el mercado y no valora aquello que se produce fuera de él, aquellos bienes y servicios que no toman la forma de valores de cambio (González, 2016).

Podemos diferenciar dos tipos de acciones que permiten la reproducción social ampliada del barrio: las cotidianas o microvecinales, y las estratégicas. Las primeras son acciones que están en el límite de lo privado y lo público y se realizan sin mucha conciencia de que aportan a la producción de un bien común; entre ellas podemos identificar la limpieza de los 
jardines familiares que dan al espacio público y de los espacios comunes, el mantenimiento de pequeñas áreas verdes, la gestión básica de los desechos (sacar la basura oportunamente), el cuidado colectivo de los niños, las acciones de ayuda solidaria y de cooperación, la provisión de espacios de recreación y ocio colectivo, el saludo y el encuentro cara a cara, la circulación de información relevante, entre otras. Las segundas - acciones estratégicas - son aquellas organizadas explícitamente en torno a un fin común, y se localizan totalmente en el espacio público. Aquí encontramos, por ejemplo, la gestión colectiva del acceso a la vivienda y su mejoramiento, el diseño y construcción de espacio público, las actividades de autoformación, la intermediación entre políticas públicas y familias, la construcción de redes con organizaciones e instituciones externas, la identificación y aprovechamiento de oportunidades políticas, el control de los compromisos públicos, el desarrollo de procesos de planificación participativo, entre otras.

Hasta 1973, la producción del espacio barrial en Chile estuvo muy vinculada a un proceso ascendente de organización vecinal, que se consagró en la Ley de Organizaciones Comunitarias del año 1968. ${ }^{4}$ El principio de reciprocidad (Polanyi-Levitt, 2014) actuaba como norma central. Sin embargo, este proceso se cortó bruscamente luego del golpe militar. Las organizaciones fueron intervenidas, se instalaron dirigentes designados y se promovió un fuerte clientelismo asociado al nuevo rol dado a las municipalidades. Pese a ello, señala Delamaza (2016), en este periodo emergieron múltiples organizaciones económicas populares y solidarias que constituyeron espacios de resistencia. Posteriormente, en el período de reconstrucción democrática, se consolidó un modelo que convirtió las organizaciones en clientes de los municipios y de los concursos públicos, cada una actuando por su lado y perdiendo la conexión entre sí, compitiendo. Esto promovió una fuerte fragmentación del tejido organizacional y, al mismo tiempo, debilitó la capacidad colectiva para abordar problemáticas de mayor escala y construir una relación simétrica con el Estado. A tal proceso se suma el descentramiento local-territorial asociado a la movilidad espacial y a las nuevas tecnologías. Con todo ello, en términos históricos, las comunidades locales han venido perdiendo la capacidad de reproducción ampliada de sus espacios barriales. Como consecuencia, el proceso de producción del barrio va hoy en el sentido contrario de la cooperación y de la idea de bien común.

En este contexto, examinaremos experiencias de gestión y articulación vecinal que tienen lugar en tres barrios de Talca, ciudad intermedia de 250.000 habitantes, capital de la región del Maule en el Valle Central de Chile. En ellas, líderes, dirigentes y vecinos, con apoyo institucional externo, como se indica en el siguiente acápite, protagonizan un proceso de fortalecimiento comunitario y de ampliación de capacidades de acción colectiva, que les permiten incrementar su poder vecinal para orientar la acción del Estado en el territorio. En ese contexto se da el Programa Territorio y Acción Colectiva. 


\section{Articulaciones vecinales para la gestión colectiva del barrio: las mesas territoriales y el Programa Territorio y Acción Colectiva}

El Programa Territorio y Acción Colectiva (TAC) es impulsado por dos ONG y una universidad regional, ${ }^{5}$ y apoya procesos de articulación vecinal en tres territorios-barrios de la ciudad de Talca desde un enfoque centrado en la Investigación Acción Participativa (IAP) ${ }^{6}$ (Letelier y Boyco, 2014). En estos territorios el TAC se ha propuesta tres objetivos. El primero, que las personas reconozcan escalas territoriales cada vez más amplias y comprendan el lugar que ocupan en ellas, lo que Ostrom denomina 'interdependencia’. Implica que los participantes salgan de su población o villa para reconocer que forman parte de un territorio más extenso que su propia población.El segundo, que se desarrollen competencias para analizar el territorio, priorizar sus problemas e identificar sus potencialidades y recursos. Finalmente, el tercero consiste en que las personas participantes desarrollen competencias para construir y negociar agendas territoriales colectivas con la autoridad, más allá de su propia villa o junta de vecinos. Así, una vez elaborada su agenda territorial, deben ser capaces de sostener un diálogo sistemático con los decisores de política, provocando una ruptura con la dicotomía top-down/bottom-up, para generar mecanismos intermedios de gestión del barrio en los cuales es la comunidad la que orienta la acción pública.

En el caso analizado, el proceso se inicia a partir de la desnaturalización/creación del territorio. Este proceso implica dos dimensiones. En una, líderes y dirigentes logran traspasar las fronteras de sus poblaciones/villas y construyen una imagen del territorio mayor al que pertenecen. Este territorio se nombra y se reconoce como un espacio de acción sobre el cual se producen procesos de territorialidad, y en él opera un proceso de articulación de escalas territoriales y organizativas. En la otra dimensión, el territorio es concebido como una construcción social en la cual han participado diversos actores, no todos con igual poder. Desde esta perspectiva, aparecen, por una parte, las políticas urbanas neoliberales y la acción de las inmobiliarias actuando conjuntamente, produciendo un negocio urbano más que un barrio. Y por otra, están las comunidades, que sin tener parte en el proceso, ocupan lo construido y tienen el desafío y la necesidad de modificarlo. Con ello surge la definición de espacios de deliberaciones colectivas, la existencia de reglas claras y de mecanismos de resolución de conflictos, así como el reconocimiento del derecho de autoorganización.

En segundo lugar, se construye un conocimiento común. Los actores desarrollan capacidades para analizar críticamente el territorio, sus problemas y potencialidades, y traducir sus malestares a un lenguaje técnicopolítico. Al mismo tiempo, son capaces de convocar a vecinos a ser parte de procesos de diagnóstico colectivo. Todo esto se ha resumido en la construcción de un saber local acerca del territorio y un relato sobre sus proble- 
mas (injusticias) y potencialidades. Lo anterior implica el comienzo de la definición del barrio y sus bienes — conectividad, transporte, espacio público, equipamiento mayor - como comunes. Todo aquello que antes estaba ahí como dado, que pertenecía a la esfera privada o pública, ahora se entiende como parte de una red de bienes sobre los cuales se puede tener cierto control.

En tercer lugar, se produce la emergencia de un "nosotros” que actúa con mayores niveles de solidaridad territorial. El grupo de dirigentes y líderes ha construido vínculos de confianza (lo que no implica ausencia de conflicto) y ha establecido marcos compartidos para la acción colectiva. Esto ha implicado ir modelando y moderando las expectativas y aspiraciones de sus integrantes respecto a sus propias poblaciones y villas, para trabajar en torno a problemáticas territoriales: accesos, espacios públicos mayores, infraestructura, equipamiento, problemas ambientales, entre otros. Al mismo tiempo, ha implicado definir colectivamente un conjunto de códigos y metodologías para cuidar las relaciones interpersonales, resolver conflictos y establecer cuotas de poder equilibradas. Un ejemplo de estos códigos es la relación entre autoridad política, territorio y el nivel menor de la población o villa. Todos entienden que cada organización poblacional tiene su agenda propia y puede libremente dialogar con el gobierno; sin embargo, se entiende al mismo tiempo que cuando se tratan temas territoriales, nadie puede "apropiarse” de la palabra para su interés específico. Cuando la Mesa Territorial negocia con la autoridad, desaparece el interés poblacional. Siguiendo a Ostrom (2011), observamos aquí procesos de concordancia de las reglas y de vigilancia respecto a ellas, creación de espacios de deliberación colectiva y mecanismos de resolución de conflictos.

Finalmente, se desarrolla la capacidad de agencia, porque a partir de un conocimiento localmente producido acerca del territorio, los sujetos logran priorizar los desafíos y establecer cursos estratégicos de acción. Su conocimiento y su capacidad de ordenar las demandas hacia el sector público han ampliado la posibilidad de interlocución y relacionamiento simétrico con la autoridad. A la vez, este "roce" con los decisores de política les ha permitido adquirir mayor comprensión de las lógicas de la acción pública, lo que les facilita moderar y procesar las expectativas en relación con los tiempos de los procesos de gestión e inversión pública. Si bien el incremento de la capacidad de orientar la acción pública no transforma el barrio en un común, sí amplía el espacio de control de la comunidad sobre el territorio y la hace emerger como un actor, allí donde antes no existía. En la medida en que esta nueva capacidad comunitaria se consolida y sobre ella se producen procesos autorreflexivos, es posible que se pase desde la participación en la gestión pública a la gestión común del barrio.

En conjunto, estos resultados apuntan a la ampliación de la capacidad de acción colectiva de las comunidades y, por tanto, abren paso al surgimiento de acciones estratégicas orientadas a lograr un control mayor en la producción del barrio. En el caso de la experiencia que analizamos, esta reproducción ampliada se expresa concretamente en al menos tres ámbitos: 
a) En el trabajo colectivo de las comunidades. Se le ha planteado a la gestión pública desafíos específicos en diversos ámbitos: seguridad vecinal, limpieza del espacio público, mejoramiento del equipamiento colectivo, acceso a información, entre otros. No es la comunidad la que gestiona el bien público directamente, pero sí es quien controla la acción del Estado para el resguardo del bien.

b) En la orientación que la comunidad ha producido en la inversión estatal de acuerdo con sus intereses y prioridades. Esto se expresa en la creación de parques, instalación de mobiliario y equipamiento comunitario, mejoramiento de espacios públicos y de las viviendas. Uno de los aspectos más interesantes en esta dimensión es que no solo se ha logrado que el Estado invierta en los territorios, sino también que lo haga de cierta manera. Cuando decimos esto no solo nos referimos a que el diseño del nuevo parque se ha hecho con participación de los vecinos, o que los nuevos paraderos se han instalado en los lugares que los propios vecinos han definido (ambas cosas efectivamente han ocurrido); también se ha producido un cambio en la lógica de la relación entre los actores públicos y las comunidades. Este cambio apunta a pasar de una situación de beneficiario pasivo de la acción pública, a la emergencia de organizaciones capaces de orientar esta acción, poner tiempos y condiciones, pero también darle insumos que aumenten su efectividad. Al respecto, las Mesas Territoriales han desempeñado un importante papel en un nuevo tipo de articulación entre comunidad y gobierno.

c) En la creación de las Mesas Territoriales. En ellas las organizaciones: i) comparten información y redes, ii) definen apoyos solidarios a causas vecinales, iii) definen y organizan acciones comunitarias y iv) hacen seguimiento a compromisos territoriales de autoridades locales y sectoriales. Poco a poco, estas Mesas también comienzan a ser espacios en los que se definen acciones comunitarias que no dependen de la acción pública, sino del esfuerzo propio de los vecinos; entre ellas, fiestas y ferias barriales, encuentros de diagnóstico participativo, campañas de denuncia pública, cuentas públicas comunitarias, etc. Este conjunto de acciones requiere poner en movimiento a diversos actores y redes de colaboración intra- y extraterritoriales, y constituye en sí mismo el inicio de una gestión colectiva del barrio. Es en esta última dimensión donde se juega la posibilidad de que la acción de los vecinos defina el barrio como un común.

\section{Reflexión: la precaria y nunca acabada construcción del barrio como un común}

En los casos de articulación vecinal que hemos revisado se observa un conjunto concertado de acciones que progresivamente van instituyendo una nueva manera de definir y relacionarse con el espacio vecinal, en la 
cual lo común surge como resultado de una praxis instituyente y genera a la vez nuevas formas de gobernanza. La cadena de acción que se observa parte de una redefinición de los límites del barrio y de la construcción de un conocimiento propio acerca de él (problemas y potencialidades). En este momento, el bien-barrio emerge como objeto social. La acción continúa con la definición de una cierta idea futura del barrio, un plan, a partir del cual los actores sociales comienzan a relacionarse con el Estado. No hablamos aquí de una gestión vecinal en la cual la propia comunidad gestione los problemas, sino de la capacidad de orientar desde abajo la acción del Estado. El barrio emerge en su dimensión de bien común, del cual debemos preocuparnos solidariamente, pero su gestión no corresponde a la propia comunidad. En este sentido, entendemos que el barrio no es un bien común per se, ya que se constituye en el encuentro entre la producción privada, la acción del Estado y la actuación de la comunidad; y adquiere su carácter a partir de sucesivas y acumulativas acciones vecinales estratégicas - las prácticas de reproducción ampliada— que comienzan a dibujar y gestionar la dimensión común.

Estamos, en este sentido, en la mitad del camino. El barrio deja de ser un bien entregado solo al mercado y deja también de ser únicamente fruto de las acciones top-down del Estado, para comenzar a ser un objeto sobre el que se pueden tener control. En este punto, la comunidad entra al ruedo de la producción del barrio. El que la comunidad comparta la producción del barrio con otras esferas no debe extrañarnos, dado que - como hemos dicho- los comunes se caracterizan por una diversidad de modos de producción y de gestión, dependientes en diversos grados de una comunidad, de los gobiernos o a veces de regímenes híbridos. La característica central del barrio es que se constituye como tal a partir de una acción colectiva que proviene de una comunidad o de una red de ciudadanos codecisores de su modo de producción o de gestión.

En el proceso a lo largo del cual los actores sociales actúan en torno al barrio y se relacionan entre ellos, van también alimentando una coactividad que se vuelve instituyente. En otras palabras, el primer esfuerzo de constituir el barrio como un objeto (redefiniendo sus límites y reconociéndolo), permite un conjunto de relaciones que van redefiniendo, simultáneamente, al objeto mismo y las relaciones sociales que permiten reproducirlo. En esta lógica se entiende que el proceso de articulación vecinal se expresa en la creación de Mesas Territoriales, y de códigos, reglas y principios de acción que permiten su funcionamiento. Aquí observamos el proceso instituyente en movimiento.

La experiencia nos muestra que, incluso en contextos adversos, es posible recuperar la dimensión comunitaria en la gestión del barrio. Al mismo tiempo, nos enseña que esa gestión, y las acciones que conlleva, van transformando las relaciones entre los sujetos, las significaciones e imaginarios del territorio y las relaciones con el Estado. Podríamos hablar de un proceso de constitución del barrio en común, en la medida en que las acciones de la comunidad transitan desde la demanda organizada al Estado, hacia procesos de cogestión cada vez más intensos. 


\section{Notas}

${ }^{1}$ Una versión anterior de este artículo ha sido publicada como parte del libro "Ensayos sobre economía cooperativa, solidaria y autogestionaria. Por una economía plural”, editado por Raúl González Meyer

${ }^{2}$ En el caso de Chile, desde finales de los años noventa se ha venido desarrollando y consolidando un conjunto de programas gubernamentales que buscan mejorar las condiciones sociales y físicas de los barrios: al respecto pueden citarse Chile Barrio, junto al ya mencionado Recuperación de Barrios (Quiero Mi Barrio), a los que se suman Regeneración de Condominios Sociales, Acción en Comunidad, Más Territorio, entre otros. Si bien ello muestra una acción pública que avanza respecto de políticas vinculadas exclusivamente a la provisión de vivienda, su fundamento político no radica en la constitución o fortalecimiento de una escala territorial para el ejercicio de la ciudadanía y la democracia, sino en la necesidad de corregir los efectos negativos de las políticas de vivienda de corte neoliberal; entre ellos, la precarización física, el debilitamiento de la confianza social y de la convivencia, la violencia urbana (Rodríguez \& Sugranyes, 2005; Ducci, 2004).

${ }^{3}$ Castoriadis propone la noción de "magma” para describir, a un nivel individual, la articulación entre el Ser y la multiplicidad de tipos de ser que lo componen; y a un nivel colectivo, a la sociedad como determinación parcial y transitoria de una indeterminación eterna (Vanhulst, 2015). El magma está definido (en términos lógicos) como “de lo que se puede extraer (o: en lo que se puede construir) organizaciones de conjunto en número indefinido, pero que nunca puede ser reconstituido (idealmente) por composición de conjunto (finita o infinita) de estas organizaciones" (Castoriadis, 1975, p. 461). Así, esta "lógica de los magmas” permite pensar la sociedad "en su elaboración”, una lógica que intenta superar las ideas de caos desorganizado y de sistemas organizados en referencia a la sociedad.

${ }^{4}$ Ley 16880, de Organizaciones Comunitarias, Ministerio del Interior, promulgada el 19 de julio de 1968; en http://www.leychile.cl/Navegar?idNorma=28688

${ }^{5}$ ONG Surmaule (Talca), Corporación SUR (Santiago) y Universidad Católica del Maule, Talca.

${ }^{6}$ La Investigación Acción Participativa (IAP), que postula como eje central el fortalecimiento de los actores sociales/comunitarios y la constante relación entre intervención y construcción de conocimiento desde las bases. Uno de los aspectos centrales de la IAP es el ciclo del conocimiento popular: la problematización de la realidad, su desnaturalización y la recuperación de la conciencia de poder sobre el territorio 


\section{Bibliografía}

Arendt, H. (1993), La condición humana, Editorial Paidós, Barcelona.

Atkinson, R., Dowling, R. \& McGuirk, P. (2009),“Home/Neighbourhood/ City/”,Environment and Planning, 41(12), 2816-2822. Doi: 10.1068/a42110

Bauman, Z. (2011), Daños colaterales. Desigualdades sociales en la era global, Fondo de Cultura Económica, Madrid.

Boyco, P., Letelier, F. \& Gualteros, J. (2016), Acción colectiva, articulación y territorio. Notas del Seminario-Encuentro ACT 2015, Talca, 12 y 13 de diciembre 2015 (pp. 4-7). Serie Aprendizajes Colectivos, N 2, Ediciones SUR, Santiago de Chile. Disponible en http://surmaule.cl/wp-content/ uploads/2016/03/APRENDIZAJES-COLECTIVS-021.pdf

Castoriadis, C. (1975), La institución imaginaria de la sociedad, Tusquets Editores, Buenos Aires.

Coraggio, J. L. (1999). Política social y economía del trabajo. Alternativas a la política neoliberal para la ciudad, Universidad Nacional de General Sarmiento / Miño y Dávila Editores, Buenos Aires.

Delamaza, G. (2016), “Una mirada a los procesos de acción colectiva en Chile”, en P. Boyco, F. Letelier \& J. Gualteros (Eds.), Acción colectiva, articulación y territorio. Notas del Seminario-Encuentro ACT 2015, Talca, 12 y 13 de diciembre 2015 (pp. 4-7). Serie Aprendizajes Colectivos, N 2. Ediciones SUR, Santiago de Chile, Disponible en http://surmaule.cl/wpcontent/uploads/2016/03/APRENDIZAJES-COLECTIVOS-021.pdf

Ducci, M. E. (2004), “Las batallas urbanas de principios del tercer milenio”, en C. de Mattos, M. E. Ducci, A. Rodríguez \& G. Yáñez Warner (Eds.), Santiago en la globalización: ¿Una nueva ciudad?, Ediciones SUR, Santiago de Chile, p. 137-166. Disponible en http://www.sitiosur.cl/r.php?id=16

González, R. (2016), “Una mirada a los procesos de acción colectiva en Chile”, en P. Boyco, F. Letelier \& J. Gualteros (Eds.), Acción colectiva, articulación y territorio. Notas del Seminario-Encuentro ACT 2015, Talca, 12 y 13 de diciembre 2015 (pp. 7-10). Serie Aprendizajes Colectivos, N², Ediciones SUR, Santiago de Chile. Disponible en http://surmaule.cl/wpcontent/uploads/2016/03/APRENDIZAJES-COLECTIVOS-021.pdf

Hardin, G. (1968), “The Tragedy of the Commons”, Science, 162(3859), 1243-1248. doi: 10.1126/science.162.3859.1243

Hirsch, F. (2005),Social Limits to Growth, Routledge and Kegan Paul, Londres.

Jackson, T. (2011), Prosperidad sin crecimiento: Economía para un planeta finito, Icaria, Barcelona. 
Polis, Revista Latinoamericana, Volumen 15, $N^{\circ}$ 45, 2016

Laval, C. \& Dardot, P. (2014), Común: Ensayo sobre la revolución en el siglo XXI, Gedisa Barcelona.

Landerretche, Ó. (2016), Vivir juntos. Economía, política y ética de lo comunitario y lo colectivo, Debate, Santiago de Chile.

Lefevbre, H. (1978), De lo rural a lo urbano, Península, Barcelona.

Letelier F. y Boyco P. (Coords.) (2014), Orientando la acción pública desde la base, Ediciones Surmaule, Talca,

Meadows, D. H., Meadows, D. L., Randers, J. \& Behrens, W. W. (1972), The limits to growth: A report for the Club of Rome's project on the predicament of mankind, Universe Books, New York.

Nussbaum, M. C. (2011), Creating Capabilities, Harvard University Press, Cambridge.

Ostrom, E. (2010), “Beyond Markets and States: Polycentric governance of complex economic systems”. Transnational Corporations Review, 2(2), 1-12. doi: 10.1080/19186444.2010.11658229

Ostrom, E. (2011), El gobierno de los bienes comunes: la evolución de las instituciones de acción colectiva, Fondo de Cultura Económica, México, DF.

Polanyi, K. (2003), La gran transformación: los orígenes políticos y económicos de nuestro tiempo. $2^{\mathrm{a}} \mathrm{ed}$. Traducción de E. L. Suárez, Fondo de Cultura Económica, México, DF. Versión de Las Ediciones de la Piqueta (Madrid, 1989)

Polanyi-Levitt, K. (2014), “Los conceptos más importantes en el trabajo de Karl Polanyi y su relevancia contemporánea” [Reseña]. Economía y Desarrollo, 151(1), 198-211. Disponible en http://www.redalyc.org/ articulo.oa?id=425541209016

Rodríguez, A. \& Sugranyes, A. (Eds.). (2005). Los con techo. Un desafío para la política de vivienda social, Ediciones SUR, Santiago de Chile. Disponible en http://www.sitiosur.cl/r.php?id=81.

Sen, A. (1999), Development as Freedom, Oxford University Press, Oxford.

Tapia, V. (2015), “¿De qué hablamos cuando hablamos de barrio? Trayectoria del concepto de barrio y apuntes para su problematización”. Antropologías del Sur, (3), 121-135. Disponible en http:// www.revistaantropologiasdelsur.cl/wp-content/uploads/2015/07/tapia_121135.pdf 
Vanhulst, J. (2015), Les chemins sinueux du développement durable. Une analyse du discours académique latino-américain. Tesis de doctorado, Universidad Libre de Bruselas, Bélgica, y Universidad Alberto Hurtado, Santiago de Chile.

Recibido: 28.10.16

Aceptado: 01.12.16 